\title{
Predicted UV-C Sensitivity of Human and Non-human Vertebrate (+) ssRNA Viruses
}

\author{
*Brahmaiah Pendyala, ${ }^{*}$ Ankit Patras \\ ${ }^{1}$ Department of Agricultural and Environmental Sciences, Food Science Program, College of \\ Agriculture, Tennessee State University, Nashville, 37209 TN USA
}

\author{
* Correspondence: \\ Co-Corresponding authors: \\ Ankit Patras, Ph.D. \\ Associate Professor \\ Email: apatras@tnstate.edu \\ Tel: 1-615-963-6007; 615-707-8436 \\ Brahmaiah Pendyala, Ph.D. \\ Research Scientist \\ Email: bpendyal@tnstate.edu \\ Tel: 1-615-963-6019/6018; 419-699-6348
}

Keywords: Genomic modeling, UV-C inactivation, human, veterinary, (+) ssRNA viruses 


\begin{abstract}
Epidemic and pandemic infectious diseases caused by RNA viruses constitute a significant hazard to human and animal health. Disinfection is an essential aspect of infection prevention and control measures. In this study, we estimated UV-C sensitivity of 83 human and veterinary pathogenic (+) ssRNA viruses by developed pyrimidine dinucleotide frequency-based genomic model. The data showed that the avian infectious bronchitis virus (genus: $\gamma$-coronavirus) with an estimated $\mathrm{D}_{90}$ value of $17.8 \mathrm{~J} / \mathrm{m}^{2}$ was highly UV sensitive, whereas Salivirus NG-J1 (genus: salivirus) with a $\mathrm{D}_{90}$ value of $346.4 \mathrm{~J} / \mathrm{m}^{2}$ was highly UV resistant. Overall, the trend of UV-C sensitivity of (+) ssRNA virus families followed as Coronaviridae $<$ Flaviviridae $<$ Togadoviridae $<$ Arteriviridae, Matonaviridae, Astroviridae $<$ Caciviridae $<$ Picornaviridae $<$ Nodaviridae $<$ Herpeviridae. The results revealed that the enveloped viral families (Coronaviridae, Flaviviridae, Togadoviridae Arteriviridae, and Matonaviridae) are more UV-C sensitive than other nonenveloped families. Further validation of the model estimated UV sensitivity with literature available experimental data showed good agreement of predicted values. The estimates presented here could make it possible to reasonably predict UV-C disinfection efficiency of human and veterinary pathogenic viruses, which need specific biosafety requirements and/or difficult to cultivate in lab conditions.
\end{abstract}




\section{Introduction}

Ten virus families whose members are pathogenic to humans and animals possess positivesense (+) single-stranded (ss) RNA genomes. The families Astroviridae, Caliciviridae, Picornaviridae, Nodaviridae and Hepeviridae are characterized by non-enveloped, whereas other families Coronaviridae, Flaviviridae, Togaviridae, Arteriviridae and Matonaviridae have enveloped capsids (https://viralzone.expasy.org/294). The spread and persistence of these pathogenic viruses in diverse environments, such as hospitals, residential, public areas, pet care facilities, animal sheds, animal husbandry, etc., emphasize developing efficient decontamination processes to control epidemic and pandemic outbreaks (Cozad and Jones, 2003). Conventional chemical decontamination procedures are time-consuming, labor and resource-intensive, prone to high degrees of human error, and not applicable to air disinfection (McGinn et al., 2020). Alternative physical disinfection methods, such as germicidal ultraviolet light treatment, have gained importance due to their potential to disinfect air (Reed, 2010) and overwhelm the limitations mentioned above (McGinn et al., 2020).

The germicidal ultraviolet light disinfection method uses UV-C light to disinfect microorganisms by damaging the nucleic acids, causing them to be unable to replicate and alters vital cellular functions (Patras et al., 2020). It is well known that the disinfection level of microorganisms by UV-C light depends on their UV susceptibility, defined as D90 or D10 (dose for $90 \%$ inactivation or $10 \%$ survival) expressed as $\mathrm{J} / \mathrm{m} 2$ or $\mathrm{mJ} / \mathrm{cm} 2$ (Patras et al., 2020). UV-C sensitivity of a wide range of microorganisms has been reported, including vegetative and spore forms of bacteria, yeast, fungi, protozoa, algae, and viruses (Malayeri et al., 2016; Gopisetty et al., 2019; Pendyala et al., 2019, 2020a, 2021). However, the UV-C sensitivity data for many (> $80 \%$ ) human and animal pathogenic viruses is not available due to the prerequisite for biosafety level 
(BSL)-3 containment and the need for specifically trained skilled labor and cultivation limitations in the laboratory environment (Pendyala et al., 2020b). Acquiring the knowledge of UV susceptibility of target viruses is essential to deliver sufficient doses for efficient decontamination of the environment.

Our earlier study developed and validated a genome-sequence-based mathematical model $\left(r^{2}=\right.$ 0.90) to predict the UV sensitivity and identify potential SARS-CoV-2 and human norovirus surrogates (Pendyala et al., 2020b). This model was developed based on the pyrimidine dinucleotides frequency (PyNNF) of genome sequence, that effects the formation of pyrimidine dimers and 6-4 photoproducts and thereby UV susceptibility. The objective of the study was to estimate the UV-C sensitivity of 83 human and veterinary pathogenic viruses, belongs to all the families of (+) ssRNA viruses (Coronaviridae, Flaviviridae, Togadoviridae, Arteriviridae, Matonaviridae, Astroviridae, Caciviridae, Picornaviridae, Nodaviridae, Herpeviridae) by using developed pyrimidine dinucleotide frequency based mathematical model. Further validation of the model-predicted data by comparison with literature available experimental data.

\section{Materials and Methods}

\section{Collection and determination of genomic parameters; genome size, and calculation of pyrimidine dinucleotide frequency value (PyNNFV)}

We collected the genomic sequence of (+) ssRNA viruses belonging to families of Flaviviridae, Picornaviridae, Arteriviridae, Coronaviridae, Togaviridae, Retroviridae, Astroviridae, Calciviridae, Nodaviridae, Hepeviridae, and Matonaviridae. The size and nucleotide sequences of genomes used in this study were directly obtained from the available NCBI genome database (Table 1-5). A novel R code was developed to count the PyNNs by the exclusive method (each pyrimidine considered in one PyNN combination only) in the order of TT > TC > CT > CC and 
considered $100 \%$ probability when PyNN are flanked by pyrimidine on both sides and $50 \%$ probability for PyNN flanked by purine on either side. Further PyNNFV values were calculated by the following equation (Pendyala et al., 2020b).

$$
\text { PyNNFV }=\frac{(\mathrm{TT} \%)(\mathrm{TC} \%)(\mathrm{CT} \%)(\mathrm{CC} \%)}{\text { Genome bp }} \quad \text { Eqn } 1
$$

\section{Estimation of UV-C sensitivity (D90) values}

Calculated PyNNF values were used to estimate the UV-C sensitivity of viruses using the reported linear regression model with $\mathrm{r}^{2}=0.90$ (Eqn 2) from our previous study (Pendyala et al., 2020b).

$$
y=(19984 \times x)+10.409 \quad \text { Eqn } 2
$$

\section{Results and discussion}

Table 1-5 depicts the selected viruses of various families of (+) ssRNA pathogenic viruses, and genus, host, diseases caused, NCBI GenBank ID, genome size, and estimated PyNNFV and D90 values. The genome size values ranged from 4528 bp to $30033 \mathrm{bp}$, and PyNNFV varied from 0.000371 to 0.016812 . Graphical representation of data shows the estimated $\mathrm{D}_{90}$ values of different virus families were in the order of Coronaviridae $<$ Flaviviridae $<$ Togadoviridae $<$ Arteriviridae, Matonaviridae, Astroviridae < Caciviridae < Picornaviridae < Nodaviridae < Hepeviridae (Figure 1). The results revealed that the enveloped virus families (Coronaviridae, Flaviviridae, Togadoviridae Arteriviridae and Matonaviridae) are more UV-C sensitive than other nonenveloped families.

\section{Elucidation of the variability of calculated UV-C sensitivity (D90) at the genus level}

Flaviviridae. The estimated UV-C sensitivity of different genera of the flaviviridae family was shown in Table 1. The data shows the genus pestivirus had higher UV-C sensitivity with $\mathrm{D}_{90}$ of 
$26.3 \mathrm{~J} / \mathrm{m}^{2}$ than flavivirus $\left(29.1-40.7 \mathrm{~J} / \mathrm{m}^{2}\right)$, pegivirus $\left(90.8 \mathrm{~J} / \mathrm{m}^{2}\right)$, and hepacivirus $(107.5-110$ $\left.\mathrm{J} / \mathrm{m}^{2}\right)$.

Picornaviridae. Table 2 shows the predicted UV-C sensitivity of various genera of the picornaviviridae family. Bluegill picornavirus belongs to limnipivirus, was predicted to be highly UV sensitive with $\mathrm{D}_{90}$ of $33.2 \mathrm{~J} / \mathrm{m}^{2}$, whereas salivirus NG-J1 was highly UV resistant $\left(346.4 \mathrm{~J} / \mathrm{m}^{2}\right)$. The results revealed that the $\mathrm{D}_{90}$ values of major genus enterovirus and genera of hepatovirus, tremovirus, sapelovirus, avihepatovirus, avisvirus, and cosavirus was in the range of $47.9-88$ $\mathrm{J} / \mathrm{m}^{2}$. Medium UV sensitivity $\left(100.9-125.7 \mathrm{~J} / \mathrm{m}^{2}\right)$ was observed with genera teschovirus, erbovirus, rosavirus, dicipivirus, and cardiovirus. Suboptimal UV resistance with D90 $_{90} 171.9$ to 267.7 J/m² was noticed with genera megrivirus, sicinivirus, kobuvirus, and sakobuvirus.

Arteriviridae and Coronaviridae. The families arteriviridae and coronaviridae are assigned to the order Nidovirales. The data show the coronaviridae family viruses were more UV sensitive $\left(\mathrm{D}_{90} 17.8-28.1 \mathrm{~J} / \mathrm{m}^{2}\right)$ than arteriviridae viruses ( $\mathrm{D}_{90} 58.8-81.2 \mathrm{~J} / \mathrm{m}^{2}$ ) (Table 3 ). In coronaviridae, genus $\gamma$-coronavirus was noticed to be more UV sensitive ( $D_{90} 17.8 \mathrm{~J} / \mathrm{m}^{2}$ ) and $\beta$-coronavirus (MERS coronavirus) was more UV resistant ( $\mathrm{D}_{90} 28.1 \mathrm{~J} / \mathrm{m}^{2}$ ). The $\lambda$-arterivirus and $\delta$-arterivirus were identified as more UV sensitive and UV resistant genera in arteriviridae.

Togadoviridae and Matonaviridae. In this family, the genus alphavirus includes mosquito-borne human and veterinary pathogenic viruses. The model predicted UV D 90 values were between 20.9 - $40.9 \mathrm{~J} / \mathrm{m}^{2}$, minimum with O'nyong-nyong virus and maximum with Venezuelan equine encephalitis virus (Table 4). Rubella virus belongs to the genus rubivirus, and family matonaviridae had predicted $\mathrm{D}_{90}$ value of $65.5 \mathrm{~J} / \mathrm{m}^{2}$.

Calciviridae. The estimated $\mathrm{D}_{90}$ of calciviridae family ranged from $65.7-98.6 \mathrm{~J} / \mathrm{m}^{2}$, and the genera lagovirus and nebovirus predicted with minimum and maximum $\mathrm{D}_{90}$ values (Table 5). The 
model predicted $\mathrm{D}_{90}$ values of human norovirus (HNoV) groups GI, GII and GIV were 69.1, 89.0, and $77.6 \mathrm{~J} / \mathrm{m}^{2}$, respectively.

Nodaviridae. This family viruses comprised of are pathogenic to fish, comprised of two genera: $\alpha$-nodavirus and $\beta$-nodavirus. The $\mathrm{D}_{90}$ data show the high resistance to UV (D90 varies from 118.4 - $156.9 \mathrm{~J} / \mathrm{m}^{2}$ ), $\alpha$-nodavirus more sensitive than $\beta$-nodavirus (Table 5).

Astroviridae. The estimated UV sensitivity of two genera, mamastrovirus and avastrovirus were 58.5 and $59.3 \mathrm{~J} / \mathrm{m}^{2}$, respectively (Table 5).

Hepeviridae. Hepatitis E virus belongs to genus hepevirus had predicted $\mathrm{D}_{90}$ value of $247.2 \mathrm{~J} / \mathrm{m}^{2}$, while other genus piscihepevirus (causes disease in fish) with lower $\mathrm{D}_{90}$ of $133.3 \mathrm{~J} / \mathrm{m}^{2}$ (Table 5).

Though the developed genomic sequence-based parameter (PyNNFV) model may be sufficient to estimate UV-C susceptibility of viruses in many scenarios, the generation of more experimental data at specific regions (where the model does not have enough empirical data) is required to improve the accuracy of our predicted $\mathrm{D}_{90}$ values. In conclusion, our model predicted D90 values could be helpful to develop an efficient UV-C treatment process to achieve the target disinfection level of specific (+) ssRNA viruses where experimental UV-C sensitivity data is not available or feasible.

Note: There are no conflicts to declare

\section{References}

Cozad, A., \& Jones, R. D. (2003). Disinfection and the prevention of infectious disease. American journal of infection control, 31(4), 243-254.

Gopisetty, V. V. S., Patras, A., Pendyala, B., Kilonzo-Nthenge, A., Ravi, R., Pokharel, B., ... \& Sasges, M. (2019). UV-C irradiation as an alternative treatment technique: Study of its 
effect on microbial inactivation, cytotoxicity, and sensory properties in cranberry-flavored water. Innovative Food Science \& Emerging Technologies, 52, 66-74.

Malayeri, A. H., Mohseni, M., Cairns, B., Bolton, J. R., Chevrefils, G., Caron, E., ... \& Linden, K. G. (2016). Fluence (UV dose) required to achieve incremental log inactivation of bacteria, protozoa, viruses and algae. IUVA News, 18(3), 4-6.

McGinn, C., Scott, R., Donnelly, N., Roberts, K. L., Bogue, M., Kiernan, C., \& Beckett, M. (2020). Exploring the Applicability of Robot-Assisted UV Disinfection in Radiology. Frontiers in Robotics and AI, 7.

Patras, A., Bhullar, M. S., Pendyala, B., \& Crapulli, F. (2020). Ultraviolet Treatment of Opaque Liquid Foods: From Theory to Practice.

Pendyala, B., Patras, A., Gopisetty, V. V. S., \& Sasges, M. (2021). UV-C inactivation of microorganisms in a highly opaque model fluid using a pilot scale ultra-thin film annular reactor: Validation of delivered dose. Journal of Food Engineering, 294, 110403.

Pendyala, B., Patras, A., Gopisetty, V. V. S., Sasges, M., \& Balamurugan, S. (2019). Inactivation of Bacillus and Clostridium spores in coconut water by ultraviolet light. Foodborne pathogens and disease, 16(10), 704-711.

Pendyala, B., Patras, A., Pokharel, B., \& D’Souza, D. (2020b). Genomic Modeling as an Approach to Identify Surrogates for Use in Experimental Validation of SARS-CoV-2 and HuNoV Inactivation by UV-C Treatment. Frontiers in Microbiology, 11.

Pendyala, B., Patras, A., Ravi, R., Gopisetty, V. V. S., \& Sasges, M. (2020a). Evaluation of UV$\mathrm{C}$ irradiation treatments on microbial safety, ascorbic acid, and volatile aromatics content of watermelon beverage. Food and Bioprocess Technology, 13(1), 101-111. 
bioRxiv preprint doi: https://doi.org/10.1101/2021.05.10.443521; this version posted May 11, 2021. The copyright holder for this preprint (which

was not certified by peer review) is the author/funder, who has granted bioRxiv a license to display the preprint in perpetuity. It is made available under aCC-BY-NC-ND 4.0 International license.

Reed, N. G. (2010). The history of ultraviolet germicidal irradiation for air disinfection. Public health reports, 125(1), 15-27.

Figure legends

Figure 1: Predicted $\mathrm{UV}_{254} \mathrm{D}_{90}$ values of (+) ssRNA vertebrate virus families 
Table 1: Genomic model predicted UV-C (254 nm) sensitivity ( $\left.\mathrm{D}_{90}\right)$ of Flaviviridae family viruses.

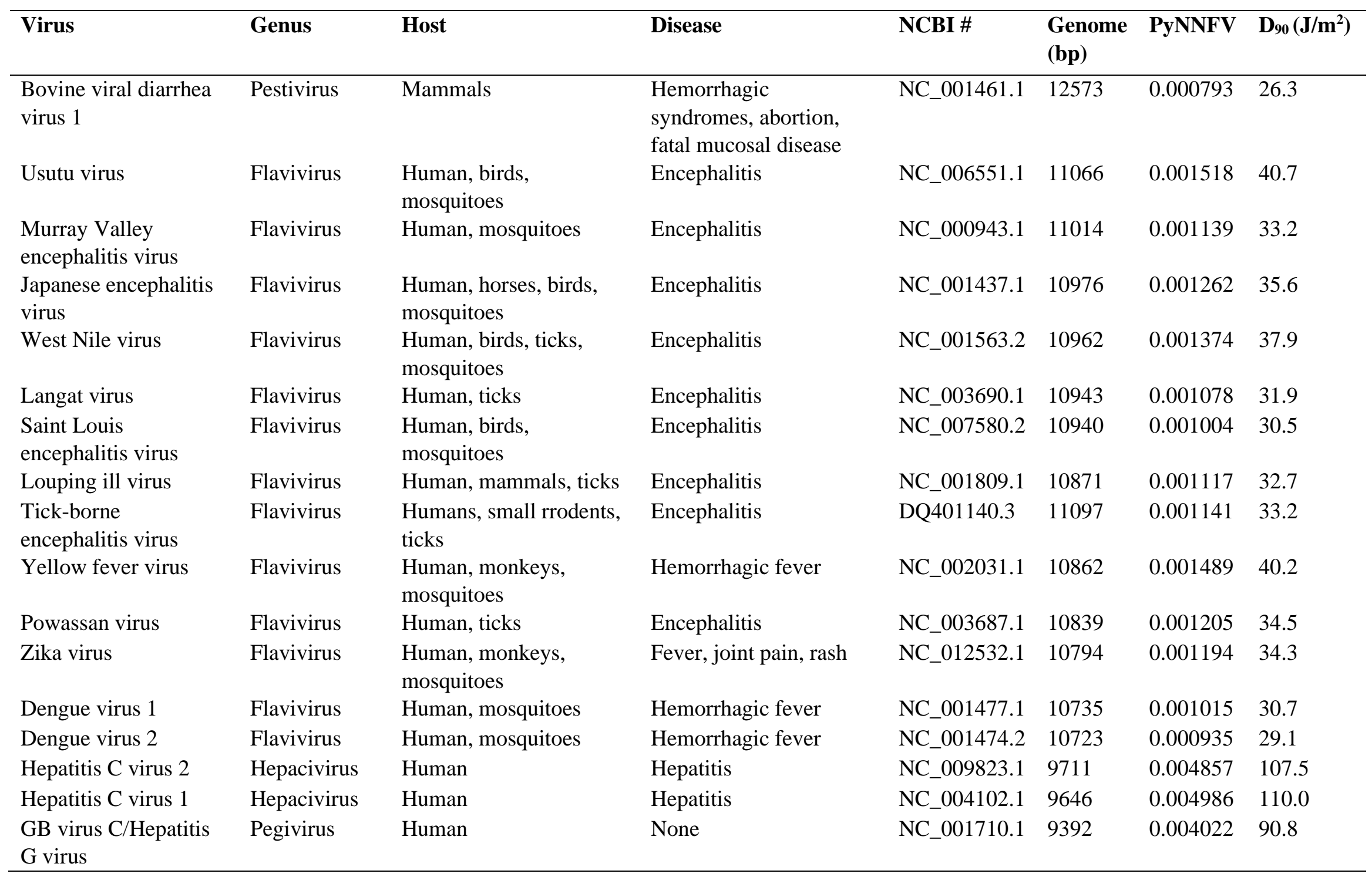


Table 2: Genomic model predicted UV-C (254 nm) sensitivity ( $\left.\mathrm{D}_{90}\right)$ of Picornaviridae family viruses.

\begin{tabular}{|c|c|c|c|c|c|c|c|}
\hline Virus & Genus & Host & Disease & NCBI \# & $\begin{array}{l}\text { Genome } \\
\text { (bp) }\end{array}$ & PyNNFV & $D_{90}\left(J / m^{2}\right)$ \\
\hline Sicinivirus 1 & Sicinivirus & Chicken, birds & - & NC_023861.1 & 9276 & 0.010280 & 215.8 \\
\hline Turkey hepatitis virus & Megrivirus & Turkey & Hepatitis & NC_021201.1 & 9075 & 0.008082 & 171.9 \\
\hline Rosavirus 2 & Rosavirus & Human & - & NC_024070.1 & 8931 & 0.005468 & 119.7 \\
\hline Equine rhinitis $B$ virus 1 & Erbovirus & Horse & - & NC_003983.1 & 8828 & 0.004771 & 105.7 \\
\hline Canine picodicistrovirus & Dicipivirus & Dog & - & NC_021178.1 & 8785 & 0.005690 & 124.1 \\
\hline Aichi virus & Kobuvirus & Human & Gastroenteritis & NC_001918.1 & 8251 & 0.012327 & 256.7 \\
\hline Bluegill picornavirus & Limnipivirus & Fish & - & NC_018506.1 & 8050 & 0.001090 & 32.2 \\
\hline Salivirus NG-J1 & Salivirus & Human & Gastroenteritis & NC_012957.1 & 7982 & 0.016812 & 346.4 \\
\hline $\begin{array}{l}\text { Encephalomyocarditis } \\
\text { virus }\end{array}$ & Cardiovirus & $\begin{array}{l}\text { Human, mouse, } \\
\text { rat, pig }\end{array}$ & Encephalitis & NC_001479.1 & 7835 & 0.005768 & 125.7 \\
\hline Human cosavirus & Cosavirus & Human & - & NC_023984.1 & 7802 & 0.003883 & 88.0 \\
\hline Duck hepatitis A virus & $\begin{array}{l}\text { Avihepato } \\
\text { virus }\end{array}$ & Ducks and geese & Fatal hepatitis & NC_008250.2 & 7711 & 0.003003 & 70.4 \\
\hline Feline sakobuvirus A & Sakobuvirus & Cat & - & NC_022802.1 & 7507 & 0.012626 & 262.7 \\
\hline Porcine sapelovirus 1 & Sapelovirus & Pig & - & NC_003987.1 & 7491 & 0.002167 & 53.7 \\
\hline Hepatitis A virus & Hepatoviruus & Human & Hepatitis & KP879217.1 & 7476 & 0.002093 & $52.2(51)$ \\
\hline Poliovirus & Enterovirus & Human & Poliomyelitis & NC_002058.3 & 7440 & 0.002626 & $62.9(73)$ \\
\hline Coxsackievirus & Enterovirus & Human & Meningitis, myo-carditis, paralysis & KX595291.1 & 7410 & 0.003138 & $73.1(79)$ \\
\hline Turkey avisivirus & Avisivirus & Turkey & - & KC614703.1 & 7373 & 0.003120 & 72.8 \\
\hline Human enterovirus 68 & Enterovirus & Human & Diarrhea, neurological disorder & NC_038308.1 & 7367 & 0.001877 & 47.9 \\
\hline Human parecho virus & Enterovirus & Human & Common cold & NC_001897.1 & 7348 & 0.002096 & $52.5(73)$ \\
\hline Human rhinovirus 14 & Enterovirus & Human & Respiratoty & NC_001490.1 & 7212 & 0.002110 & 52.6 \\
\hline Porcine teschovirus 1 & Teschovirus & Pig & Teschen disease & NC_003985.1 & 7117 & 0.004529 & 100.9 \\
\hline Human rhinovirus $\mathrm{C}$ & Enterovirus & Human & Respiratoty & NC_009996.1 & 7099 & 0.002872 & 67.8 \\
\hline $\mathrm{AEV}$ & Tremovirus & Birds & $\begin{array}{l}\text { Reduced hatching. Tremors and/or } \\
\text { ataxia. }\end{array}$ & NC_003990.1 & 7055 & 0.002126 & 52.9 \\
\hline Swine pasivirus 1 & Pasivirus & Pig & - & NC_018226.1 & 6916 & 0.003108 & 72.5 \\
\hline
\end{tabular}

Notes: $\mathrm{D}_{90}$ in parenthesis refers literature experimental values (Pendyala et al., 2020); AEV - Avian encephalomyelitis virus) 
Table 3: Genomic model predicted UV-C (254 nm) sensitivity ( $\left.\mathrm{D}_{90}\right)$ of Arteriviridae and Coronaviridae family viruses.

\begin{tabular}{|c|c|c|c|c|c|c|c|}
\hline Virus & Genus & Host & Disease & NCBI \# & $\begin{array}{l}\text { Genome } \\
\text { (bp) }\end{array}$ & PyNNFV & $D_{90}\left(J / m^{2}\right)$ \\
\hline Equine arteritis virus & $\alpha$-arterivirus & Horse & $\begin{array}{l}\text { vascular lesions, fever, edema, } \\
\text { abortion }\end{array}$ & NC_002532.2 & 12704 & 0.003112 & 72.6 \\
\hline Lelystad virus & $\beta$-arterivirus & Pig & Abortions and respiratory disease & M96262.2 & 15111 & 0.003014 & 70.6 \\
\hline $\begin{array}{l}\text { Simian hemorrhagic } \\
\text { fever virus }\end{array}$ & $\delta$-arterivirus & Monkey & $\begin{array}{l}\text { Fever, edema, dehydration, } \\
\text { hemorrhages, death (almost 100\%) }\end{array}$ & NC_003092.2 & 15717 & 0.003541 & 81.2 \\
\hline $\begin{array}{l}\text { Forest pouched giant rat } \\
\text { arterivirus }\end{array}$ & $\lambda$-arterivirus & Rat & - & NC_026439.1 & 14953 & 0.002423 & 58.8 \\
\hline $\begin{array}{l}\text { Wobbly possum disease } \\
\text { virus }\end{array}$ & $\kappa$-arterivirus & Brushtail possum & - & NC_026811.2 & 12917 & 0.002640 & 63.2 \\
\hline $\begin{array}{l}\text { Human coronavirus } \\
229 \mathrm{E}\end{array}$ & $\alpha$-coronavirus & Human & Respiratoty & NC_002645.1 & 27317 & 0.000489 & 20.2 \\
\hline SARS coronavirus 1 & $\beta$-coronavirus & $\begin{array}{l}\text { Human, bats, } \\
\text { palm civet }\end{array}$ & Respiratoty & NC_004718.3 & 29751 & 0.000674 & 23.9 \\
\hline SARS coronavirus 2 & $\beta$-coronavirus & $\begin{array}{l}\text { Human, bats, } \\
\text { pangolin? }\end{array}$ & Covid-19 & MT192772.1 & 29891 & 0.000555 & 21.5 \\
\hline MERS coronavirus & $\beta$-coronavirus & $\begin{array}{l}\text { Human, Tomb } \\
\text { bat }\end{array}$ & Respiratoty & MH734115.1 & 30033 & 0.000883 & 28.1 \\
\hline $\begin{array}{l}\text { Thrush coronavirus } \\
\text { HKU12-600 }\end{array}$ & $\delta$-coronavirus & $\begin{array}{l}\text { grey-backed } \\
\text { Thrush }\end{array}$ & Respiratory & NC_011549.1 & 26396 & 0.000690 & 24.2 \\
\hline $\begin{array}{l}\text { Avian infectious } \\
\text { bronchitis virus }\end{array}$ & $\gamma$-coronavirus & $\begin{array}{l}\text { Chicken and } \\
\text { turkey }\end{array}$ & Respiratoty & NC_001451.1 & 27608 & 0.000371 & 17.8 \\
\hline $\begin{array}{l}\text { White bream virus (Toro } \\
\text { virinae (subfamily) }\end{array}$ & Bafinivirus & Fish & $\begin{array}{l}\text { Hemorrhagic liver with necrotic } \\
\text { areas, splenomegaly, enteritis }\end{array}$ & NC_008516.1 & 26660 & 0.000954 & 29.5 \\
\hline $\begin{array}{l}\text { Breda virus (Toro- } \\
\text { virinae (subfamily) }\end{array}$ & Torovirus, & $\begin{array}{l}\text { Human, cattle, } \\
\text { pig, horse }\end{array}$ & Gastroenteritis & NC_007447.1 & 28475 & 0.000517 & 20.7 \\
\hline
\end{tabular}


Table 4: Genomic model predicted UV-C $(254 \mathrm{~nm})$ sensitivity $\left(\mathrm{D}_{90}\right)$ of Togaviridaeae and Matonaviridae family viruses.

\begin{tabular}{|c|c|c|c|c|c|c|c|}
\hline Virus & Genus & Host & Disease & NCBI \# & $\begin{array}{l}\text { Genome } \\
\text { (bp) }\end{array}$ & PyNNFV & $D_{90}\left(J / m^{2}\right)$ \\
\hline O'nyong-nyong virus & Alphavirus & $\begin{array}{l}\text { Human, } \\
\text { mosquitoes }\end{array}$ & Fever, joint pain & NC_001512.1 & 11835 & 0.000930 & 29.0 \\
\hline Chikungunya virus & Alphavirus & $\begin{array}{l}\text { Human, } \\
\text { monkeys, } \\
\text { mosquitoes }\end{array}$ & Fever, joint pain & NC_004162.2 & 11826 & 0.001108 & 32.6 \\
\hline $\begin{array}{l}\text { Eastern equine } \\
\text { encephalitis virus }\end{array}$ & Alphavirus & $\begin{array}{l}\text { Human, birds, } \\
\text { mosquitoes }\end{array}$ & Encephalitis & NC_003899.1 & 11675 & 0.001470 & 39.8 \\
\hline Ross River virus & Alphavirus & $\begin{array}{l}\text { Human, } \\
\text { mosquitoes, } \\
\text { marsupials. }\end{array}$ & Fever, joint pain & NC_001544.1 & 11657 & 0.001187 & 34.1 \\
\hline Barmah Forest virus & Alphavirus & $\begin{array}{l}\text { Human, } \\
\text { marsupials, } \\
\text { mosquitoes }\end{array}$ & Fever, joint pain & NC_001786.1 & 11488 & 0.001194 & 34.3 \\
\hline Mayaro virus & Alphavirus & $\begin{array}{l}\text { Human, } \\
\text { mosquitoes }\end{array}$ & Fever, joint pain & NC_003417.1 & 11411 & 0.001518 & 40.8 \\
\hline Sindbis virus & Alphavirus & $\begin{array}{l}\text { Human, birds, } \\
\text { mosquitoes }\end{array}$ & Pogosta disease, Fever, joint pain & NC_001547.1 & 11703 & 0.001486 & $40.1(55)$ \\
\hline $\begin{array}{l}\text { Venezuelan equine } \\
\text { encephalitis virus }\end{array}$ & Alphavirus & $\begin{array}{l}\text { Human, rodents, } \\
\text { mosquitoes }\end{array}$ & Fever, joint pain & NC_001449.1 & 11444 & 0.001525 & $40.9(55)$ \\
\hline $\begin{array}{l}\text { Western equine } \\
\text { encephalitis virus }\end{array}$ & Alphavirus & $\begin{array}{l}\text { Human, } \\
\text { vertebrates, } \\
\text { mosquitoes }\end{array}$ & Fever, joint pain & NC_003908.1 & 11484 & 0.001512 & $40.7(54)$ \\
\hline Rubella virus & Rubivirus & Human & Rubella & NC_001545.2 & 9762 & 0.002757 & 65.5 \\
\hline
\end{tabular}

Notes: D90 $_{90}$ in parenthesis refers literature experimental values (Pendyala et al., 2020) 
Table 5: Genomic model predicted UV-C $(254 \mathrm{~nm})$ sensitivity $\left(\mathrm{D}_{90}\right)$ of Astroviridae, Calciviridae, Nodaviridae, and Hepeviridae family viruses.

\begin{tabular}{|c|c|c|c|c|c|c|c|}
\hline Virus & Genus & Host & Disease & NCBI \# & $\begin{array}{l}\text { Genome } \\
\text { (bp) }\end{array}$ & PyNNFV & $D_{90}\left(J / m^{2}\right)$ \\
\hline Human astrovirus & $\begin{array}{l}\text { Mamastro } \\
\text { virus }\end{array}$ & $\begin{array}{l}\text { Human, } \\
\text { mammals }\end{array}$ & Gastroenteritis & NC_001943.1 & 6813 & 0.002446 & 59.3 \\
\hline Avastrovirus 1 & Avastrovirus & Turkey, birds & $\begin{array}{l}\text { Gastroenteritis, liver, or kidney } \\
\text { damages }\end{array}$ & Y15936.2 & 7003 & 0.002405 & 58.5 \\
\hline Human norovirus GI & Norovirus & Human & Gastroenteritis & NC_001959.2 & 7654 & 0.002936 & 69.1 \\
\hline Human norovirus GII & Norovirus & Human & Gastroenteritis & KF712510.1 & 7509 & 0.003934 & 89.0 \\
\hline Human norovirus GIV & Norovirus & Human & Gastroenteritis & JF781268.1 & 7839 & 0.003360 & 77.6 \\
\hline Sapovirus & Sapovirus & Human & Gastroenteritis & NC_006554.1 & 7476 & 0.003672 & 83.8 \\
\hline $\begin{array}{l}\text { Rabbit hemorrhagic } \\
\text { disease virus-FRG }\end{array}$ & Lagovirus & Lagomorphs & $\begin{array}{l}\text { Necrotizing hepatitis leading to } \\
\text { fatal hemorrhages }\end{array}$ & NC_001543.1 & 7437 & 0.002764 & 65.7 \\
\hline Newbury agent 1 virus & Nebovirus & Bovine & Necrotizing hepatitis & NC_007916.1 & 7454 & 0.004414 & 98.6 \\
\hline Feline calicivirus & Vesivirus & Feline & Conjunctivitis, respiratory disease & NC_001481.2 & 7683 & 0.003627 & $82.9(60)$ \\
\hline $\begin{array}{l}\text { Vesicular exanthema } \\
\text { of swine virus }\end{array}$ & Vesivirus & $\begin{array}{l}\text { Swine, sea } \\
\text { mammals }\end{array}$ & Respiratory disease & NC_002551.1 & 8284 & 0.003508 & 80.5 \\
\hline Nodamura virus & $\alpha$-nodavirus & Mammals, fishes & Paralysis and death & $\begin{array}{l}\text { AF174533.1, } \\
\text { AF174534.1 }\end{array}$ & 4540 & 0.005406 & 118.4 \\
\hline $\begin{array}{l}\text { Striped jack nervous } \\
\text { necrosis virus }\end{array}$ & $\beta$-nodavirus & Fish & $\begin{array}{l}\text { Viral encephalopathy and } \\
\text { retinopathy }\end{array}$ & $\begin{array}{l}\text { NC_003448.1, } \\
\text { NC_003449.1 }\end{array}$ & 4528 & 0.007331 & 156.9 \\
\hline $\begin{array}{l}\text { Dragon grouper } \\
\text { nervous necrosis virus }\end{array}$ & $\beta$-nodavirus & Fish & Viral nervous necrosis & AY721616.1 & 4536 & 0.006953 & 149.4 \\
\hline Barfin flounder virus & $\beta$-nodavirus & Fish & Viral nervous necrosis & EU826137.1 & 4533 & 0.006839 & 147.1 \\
\hline Hepatitis E virus & Hepevirus & $\begin{array}{l}\text { Human, pig, } \\
\text { monkey, chicken }\end{array}$ & Hepatitis & NC_001434.1 & 7176 & 0.011850 & 247.2 \\
\hline Cutthroat trout virus & Piscihepevirus & Fish & - & NC_015521.1 & 7410 & 0.006150 & 133.3 \\
\hline
\end{tabular}

Notes: D $_{90}$ in parenthesis refers literature experimental values (Pendyala et al., 2020) 
bioRxiv preprint doi: https://doi.org/10.1101/2021.05.10.443521; this version posted May 11, 2021. The copyright holder for this preprint (which

was not certified by peer review) is the author/funder, who has granted bioRxiv a license to display the preprint in perpetuity. It is made available under aCC-BY-NC-ND 4.0 International license.

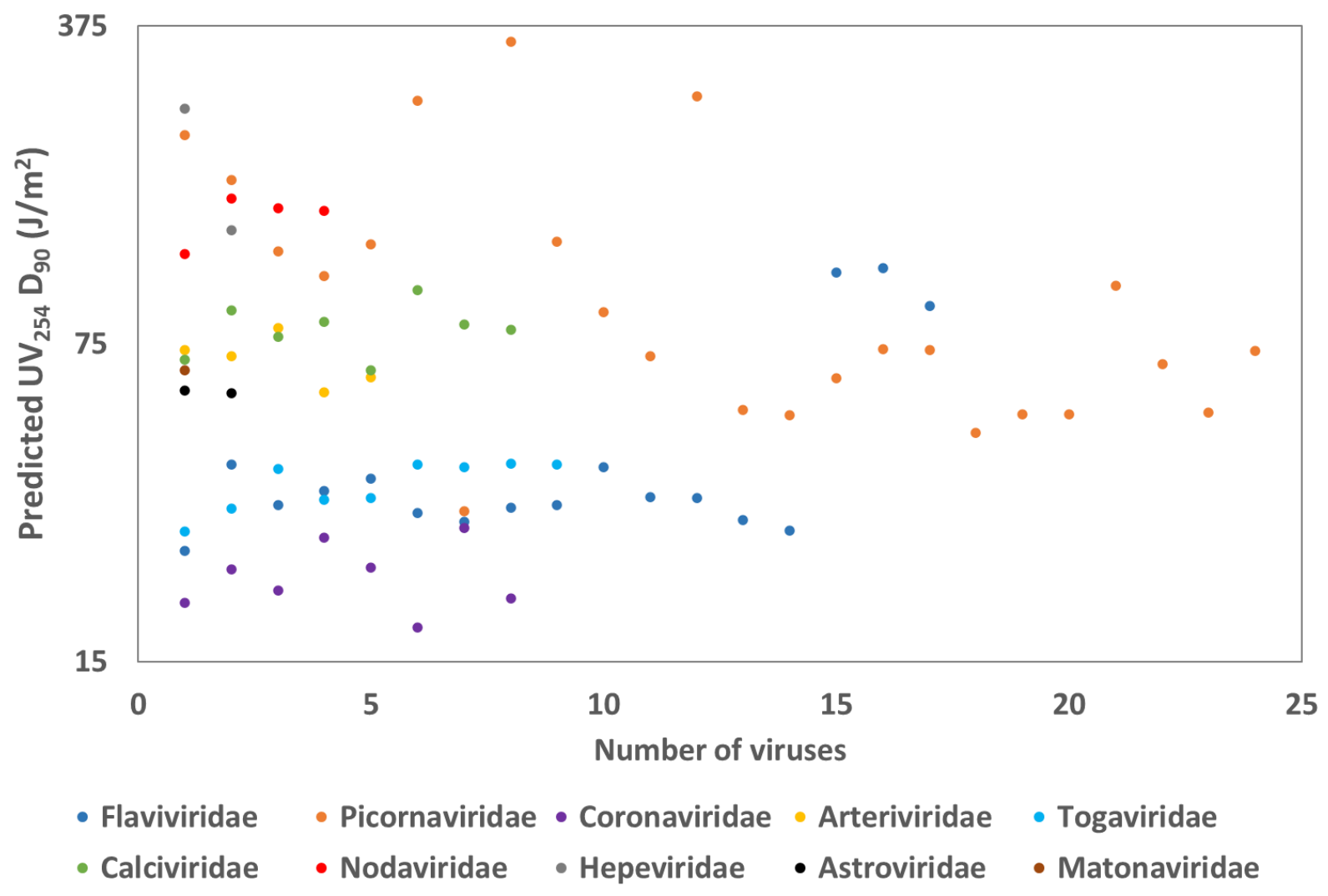

Figure 1: Predicted $U_{254} D_{90}$ values of (+) ssRNA vertebrate virus families 